\title{
HOT mutation screening in human glioblastomas
}

\begin{abstract}
Aims: Somatic mutations in IDH1 and IDH2 are described in glioblastomas (GBMs). Mutant IDH1 and IDH2 reduce $\alpha-K G$ to D-2HG which accumulates, and is proposed to promote tumorigenesis. HOT catalyzes the conversion of $\gamma$-hydroxybutyrate to succinic semialdehyde in a reaction that produces D-2HG. Since increased HOT enzyme activity could lead to an accumulation of D-2HG, coupled with the fact that only a minority of GBMs carry IDH1/2 mutations and 2HG accumulation has recently been described in IDH wild-type tumors, we analyzed a set of GBM samples for mutations in the HOT gene. Materials \& methods: We screened 42 human GBM samples for mutations in HOT. Results: No mutations in HOT were identified in the 42 GBM samples screened. Conclusion: Mutations in the coding regions of HOT do not occur at an appreciable frequency in GBM.
\end{abstract}

Genetic changes in genes called IDH have been shown to occur regularly in brain tumors. These changes result in the production of a chemical called D-2HG which accumulates to a high level in cells and is thought to damage normal cells, causing them to become cancer cells. Genetic changes in other genes may also result in the production of D-2HG and cause cancer in the same way as changes in IDH do. One such gene is called HOT. This study investigated whether genetic changes in HOT could be found in brain tumors.

Keywords: $\alpha$-ketoglutarate $\bullet$ 2-hydroxyglutarate $\bullet$ brain $\bullet$ glioblastoma $\bullet$ glioma

- hydroxyacid-oxoacid transhydrogenase $\bullet$ isocitrate dehydrogenase $\bullet$ metabolism $\bullet$ mutation

Gliomas are a diverse group of tumors with respect to morphology, genetic status and prognosis and account for $70 \%$ of all primary CNS neoplasms. WHO grade II and III gliomas are invasive and typically progress to higher-grade lesions with poor outcome. Glioblastoma (GBM) represents the commonest glioma and may develop in the absence of a less malignant precursor (primary GBM), or through progression from a lower-grade tumor (secondary GBM). GBM carries a very poor prognosis [1,2].

Somatic mutations in genes encoding the enzymes IDH1 and IDH 2 have been identified through genome-wide sequencing studies in approximately $70 \%$ of grade II and III gliomas, and in approximately $5 \%$ of
GBMs [3,4], (for a review see [5]). These mutations are early events in gliomagenesis [6], which are associated with a relatively favorable prognosis [7], and may be clinically useful as diagnostic $[8,9]$ and predictive biomarkers [10], as well as potential therapeutic targets in the future [11]. Mutations in IDH1/2 also occur in patients with acute myeloid leukemia (AML) [12], angioimmunoblastic T-cell lymphoma (AITL) [13] and other solid tumors [14-19].

IDH1 and IDH2 are homodimeric enzymes that catalyze the oxidative decarboxylation of isocitrate to $\alpha-K G$ and reduce NADP to NADPH. IDH1 is localized to the cytoplasm and peroxisome [20], where it serves as a major source of cytosolic NADPH, plays
Daniel Krell*,1,2, Paul Mulholland ${ }^{2}$, Justin Stebbing ${ }^{3}$, Ian Tomlinson ${ }^{1}$ \& Chiara Bardella ${ }^{1}$ ${ }^{1}$ Molecular \& Population Genetics Laboratory, Wellcome Trust Center for Human Genetics, University of Oxford, Oxford, UK

${ }^{2}$ Department of Medical Oncology, University College London Hospitals, London, UK

${ }^{3}$ Division of Oncology, Department of Surgery \& Cancer, Imperial Center for Translational \& Experimental Medicine, Imperial College, Hammersmith Hospital Campus, Du Cane Road, London, W12 ONN, UK

*Author for correspondence: Tel.: +44 07939264615 danielkrell@nhs.net 
a role in cellular metabolic processes [21,22], and protects against oxidative stresses [23]. IDH2 is localized to the mitochondrion where its main function is to regulate the tricarboxylic acid cycle [20]. A third enzyme, IDH3, is also localized to the mitochondrion and plays a key role in tricarboxylic acid cycle function.

Pathogenic mutations in IDHI and IDH2 are heterozygous somatic missense changes that almost always occur at a single, specific residue at the enzyme active site. Mutations in IDH1 generally affect arginine 132 (R132) [3], which is commonly substituted for histidine $(\mathrm{R} 132 \mathrm{H})$, but mutations of R132 to serine, cysteine, glycine, leucine and lysine have also been identified [4,24]. Moreover, mutations in IDHI occurring at arginine 100 (R100), which may also be pathogenic [19,24-25] have also been identified in gliomas. Mutations in IDH2 affect arginine 172 (R172) and arginine 140 (R140), the former being analogous to IDH1 R132 and the latter to IDH1 R100 [4,26]. Mutations in the active site arginine residues possess neoenzymatic activity, which results in the reduction of $\alpha-K G$ to D-2HG $[19,26-28]$, which accumulates at high levels in IDH1- and IDH2-mutated tumors [26,27]. It has been stated that D-2HG acts as an oncometabolite, with numerous potential protumorigenic effects, most likely as a result of its ability to competitively inhibit $\alpha$-KG-dependent enzymes [29,30]. Moreover, a direct correlation between the amount of $2 \mathrm{HG}$ produced by mutant $I D H 1 / 2$ and the characteristics of the resulting tumor seems to exist, since patients with $I D H 2$ R140-mutant AML produce lower levels of D-2HG and have a better prognosis those with IDH2 R172 mutations [19].

Out of the 60 or more, $\alpha$-KG-dependent enzymes in human cells, particular interest has centered on the inhibition by D-2HG of HIF-PHD, the Jumonji$\mathrm{C}$ domain containing histone demethylases, the Ten-Eleven Translocation family of 5-methylcytosine hydroxylases and collagen prolyl 4-hydroxlase. Inhibition of these enzymes could lead to tumor formation by causing HIF1 $\alpha$ target gene overexpression [29,31-32], histone modifications [29,33], aberrant DNA methylation [29-30,34-36] and abnormal collagen maturation [29,32], respectively.

D-2HG normally functions as an intermediate in the production of 5-aminolevulinate and porphyrin in heme synthesis $[37,38]$. Our current understanding of the cellular metabolism of D-2HG is limited, but two enzymes, D-2HGDH and HOT are known to affect its cellular levels [39,40]. In fact, early studies performed on mitochondrial fraction of rat kidney, liver and brain demonstrated that D-2HG is produced from the activity of HOT, which catalyzes the conversion of GHB to SSA, with a stoichiometric production of
D-2HG from $\alpha-K G$ [41], (for a review see [42]), and the existence of HOT in humans was then demonstrated in homogenates of human liver, lymphoblast and fibroblasts [43,44]. D-2HG produced by HOT is subsequently interconverted to $\alpha-K G$ via the activity of $\mathrm{D}-2 \mathrm{HGDH}$, in order to maintain carbon balance.

Homozygous inactivating mutations in $\mathrm{D}-2 \mathrm{HGDH}$ lead to D-2HG accumulation in tissues, resulting in the neurological condition hereditary D-2-hydroxyglutaric aciduria (D-2-HGA) [43]. D-2-HGA is associated with developmental delay, hypotonia and seizures, but not an increased risk of brain tumors. Conversely, L-2-hydroxyglutaric aciduria, which occurs due to inactivating mutations in $L-2 H G D H$, is weakly associated with the development of brain tumors [45]. Germline IDH2 mutations affecting arginine 140 , have also been reported in patients with D-2-HGA carrying wild-type (WT) $D 2 H G D H$ [46]. However, we and others have not identified $D 2 H G D H$ mutations in gliomas [47,48]. Mass isotopomer studies in patients with the D-2-HGA have demonstrated that accumulated D-2HG derives from mitochondrial $\alpha-K G$ [49], and it has been suggested that alterations in HOT activity may be involved in the excess D-2HG production observed [41,44,50-51]. However enzyme activity assays in fibroblast homogenates from patients with D-2-HGA demonstrated normal HOT activity [44].

Nevertheless, potential evidence for the ability of increased HOT activity to result in excess D-2HG production comes from the observation of patients with the hereditary disorders SSADH deficiency [52], and $\alpha$-ketoglutarate dehydrogenase deficiency [53]. In addition to GHB, considerably increased levels of D2HG were observed in body fluids of some patients affected by SSADH deficiency, and consequently in this disease elevated intracellular GHB levels are thought to drive HOT enzyme activity to produce secondary increased levels of D2HG [52]. Interestingly, D2HG also accumulates in the brain, liver and kidney of SSADH-deficient mice, showing a consistent link between SSADH deficiency and D2HG accumulation [54]. In patients with $\alpha$-ketoglutarate dehydrogenase deficiency accumulated $\alpha-K G$ is thought to alter the kinetic equilibrium of HOT [53] and may provide an explanation for the elevated urinary $2 \mathrm{HG}$ levels observed in this condition $[55,56]$. Furthermore, siRNA directed inhibition of HOT function has been shown to reduce cellular 2HG levels in vitro [57].

Interestingly, 2HG has recently been shown to accumulate at high levels in a subset of IDH WT gliomas, using magnetic resonance spectroscopy [58]. Furthermore, 2HG has been shown to accumulate at millimolar concentrations in IDH WT breast cancer tissues, in association with $M Y C$ gene expression [57]. 
These findings raise the possibility that mechanisms of $2 \mathrm{HG}$ accumulation unrelated to $I D H$ mutation may exist in gliomas.

Since an increase in HOT enzyme activity could lead to an accumulation of D-2HG, with tumorigenic effects similar to those observed in tumors with mutant IDH1/2 and 2HG has recently been shown to accumulate at high concentrations in IDH WT tumors, coupled with the fact that only a minority of GBMs carry IDH1/2 mutations, we analyzed a set of GBM samples for mutations in the HOT gene.

\section{Materials \& methods}

\section{Sample collection}

All brain tumors were obtained from the neuropathology department at The Royal Free Hospital, Hampstead, London. All 42 samples analyzed were confirmed to be WHO grade IV glioblastoma. We have worked solely on anonymous samples. Study of these has been approved by Oxfordshire REC B 05/Q1605/66.

\section{DNA extraction}

DNA was extracted from paraffin embedded samples using DNeasy Blood and Tissue from QuiagenR (CA, USA), following the manufacturer's instructions.

\section{Sequencing analysis}

Mutation screening of each gene was performed by direct sequencing of genomic DNA in forward and reverse orientations using the Applied Biosystems BigDye terminator reaction kit and the $\mathrm{AB} 3730 \mathrm{xl}$ sequencing machine (Applied Biosystems, CA, USA). Primer sequences were designed to encompass all
14 exons of HOT (RefGene:NM_144650). Primer sequences are illustrated in Table 1. PCR conditions are available on request.

\section{Results}

We screened 42 primary glioblastomas (WHO grade IV) for mutations in the 14 exons of HOT. Four of these tumors were known from previous analysis to carry IDH1 R132H mutations, but none carried mutations in $I D H 2, I D H 3, L 2 H G D H$ or $D 2 H G D H[47]$. No somatic mutations were identified in HOT. One previously described missense single nucleotide polymorphism (GCC->GTC) was identified in exon 1 of HOT.

\section{Conclusion \& future perspective}

We conclude that mutations in the coding regions of $H O T$ do not occur at an appreciable frequency in IDH WT GBM. The fact that $I D H$ mutations occur more frequently in grade II and grade III glioma than in GBM raises the possibility that $H O T$ mutations may also be identified in earlier grade tumors than those included in this study.

It is also feasible, however, that although mutations in the coding region of HOT were not identified in the IDH WT GBM patients who were studied, other genetic changes with the potential to increase HOT activity might exist in these tumors. For example, copy number variations encompassing HOT could occur, thus increasing gene expression. Indeed interestingly, several large copy number variations have been described encompassing the HOT gene, including one linked to AML [59]. Alternatively, mutations in possibly uncharacterized regulatory regions of HOT, such as

\begin{tabular}{|c|c|c|}
\hline Exon & Forward primer & Reverse primer \\
\hline 1 & TGGCTTGAGGCTTAGACAGG & AAGACTGCGCAGGATCTGA \\
\hline 2 & TGCCAAGAACTACCAATTTGA & GCCAAATGCAGAAACCAAAA \\
\hline 3 & TGTTCACTGCTGGATTTTACTATCA & TGGTCTGATCAAATAGAGATTATGGA \\
\hline 4 & ATGGACTGGCCAACTCTCAA & CTTCTGGCAAACTCACGTTG \\
\hline 5 & GCAGTTCTAGTGGAGTTGAACCTA & GCTTCTGCTTAAAGTAAAGAAACAA \\
\hline 6 & GGCATGGCAATTTATTTCTGA & CTCATTGCAAAATAAAACAAACA \\
\hline 7 & TTTCСTTCTCTAАTTTTGTTCСTCA & TGGAGTTGAAAGGTAAGGGAGA \\
\hline 8 & AATGATGCCCATGGCTTTAG & TGTGCGTTGAGCAACAAAAT \\
\hline 9 & GAGGAAGATGACGCTTTCCA & TGTGGGCCTCCTGTTCTATT \\
\hline 10 & AGGAATTGCCAATGTTGATG & AАATTTTACCACCTGTCCTGAA \\
\hline 11 & GCATCCCCATAAGAGTATCTTTC & TCTAAGCAGGCGAGAATTGG \\
\hline 12 & CAGGGAAGAACAGCTTTGTCA & CTCCCAAAATGCTGGGATTA \\
\hline 13 & AGAGCCCGTTTCTTCCTCTC & CTCCTTCGCAGGGAGTGA \\
\hline 14 & СTTTTCAAAGCCCTGGGTAA & TCAGCTCTTGCTCTCAGCAC \\
\hline
\end{tabular}


the binding sequence for transcriptional repressors or activators, or epigenetic changes such as alterations of promoter methylation could occur and result in an increase in $H O T$ transcriptional activity. Interestingly, promoter hypermethylation affecting HOT transcriptional activity has been identified in colorectal cancer, although in this case, this led to a downregulation in HOT expression [60].

It remains to be seen if mechanisms of $2 \mathrm{HG}$ accumulation unrelated to $I D H$ mutation exist in gliomas. However, in view of the fact that $2 \mathrm{HG}$ has recently been shown to accumulate in IDH WT tumors, additional studies are required to identify pathogenic mutations in $H O T$ and other candidate genes such as $M Y C$, which may have the potential to increase $\mathrm{D}-2 \mathrm{HG}$ production and result in cellular effects similar to those seen in IDH1 and IDH2 mutant tumors.

Therapies targeting mutant IDH1 and IDH2 have been developed, which have been shown to block D-2HG production and to reverse some of the cellular effects associated with mutant $I D H$ expression [61,62]. Furthermore, the targeted inactivation of IDH1 R132H by the inhibitor AGI-5198 has been shown to impair the growth of IDH1-mutant glioma cells and mouse xenografts, and promote gliogenic differentiation [63]. Although the potential clinical benefit of such inhibitors is not yet clear, their emergence raises new hope for the treatment of $I D H$-mutated tumors. If mutations in genes other than $I D H I$ and $I D H 2$ are indeed discovered to have the potential to increase cellular D-2HG levels, with protumorigenic effects, then such mutations may also serve as potential therapeutic targets in the future.

\section{Financial \& competing interests disclosure}

This work was supported by the charity: Mothers and Daughters, the University College London Hospitals, Experimental Cancer Medicine Center, a grant from CRUK (C6199/ A16459) and a core center grant from the Wellcome Trust (075491/Z/04). The authors have no other relevant affiliations or financial involvement with any organization or entity with a financial interest in or financial conflict with the subject matter or materials discussed in the manuscript apart from those disclosed.

No writing assistance was utilized in the production of this manuscript.

\section{Ethical conduct of research}

The authors state that they have obtained appropriate institutional review board approval or have followed the principles outlined in the Declaration of Helsinki for all human or animal experimental investigations. In addition, for investigations involving human subjects, informed consent has been obtained from the participants involved.

\section{Open Access}

This work is licensed under the Creative Commons Attribution 4.0 License. To view a copy of this license, visit http://creativecommons.org/licenses/by/4.0/

\section{Executive summary}

- Somatic mutations in IDH1 and IDH2 occur in grade II and III gliomas, and primary and secondary glioblastomas (GBMs).

- Mutant IDH1 and IDH2 exhibit novel enzymatic activity, reducing $\alpha-K G$ to D-2HG, which accumulates.

- $\mathrm{D}-2 \mathrm{HG}$ is thought to act as an oncometabolite through its inhibition of $\alpha-K G$ dependent enzymes.

- $2 \mathrm{HG}$ has recently been shown to accumulate in IDH wild-type tumors.

- HOT catalyzes the conversion of GHB to SSA in a reaction that converts $\alpha-K G$ to D-2HG.

- Mutations in HOT could also therefore potentially lead to D-2HG accumulation and thus to tumorigenesis via mechanisms similar to those associated with mutant IDH1/2.

- We screened 42 human GBM samples for mutations in the coding regions of $H O T$, but none were identified.

- Hence, we conclude that mutations in the coding region of HOT do not occur at an appreciable frequency in GBM.

- However, somatic copy number alterations and epigenetic changes such as altered promoter methylation may also have the potential to alter HOT activity in glioma.

- Additional studies are warranted to identify pathogenic mutations in HOT and other genes which may have the potential to increase D-2HG production and result in cellular effects similar to those seen in IDH1 and IDH2 mutant tumors.

\section{References}

Papers of special note have been highlighted as:

- of interest

1 Ohgaki H. Epidemiology of brain tumours. Methods Mol. Biol. 472, 323-342 (2009).

2 Wen PY, Kesari S. Malignant gliomas in adults. N. Engl. J. Med. 359, 492-507 (2008).
3 Parsons DW, Jones S, Zhang X et al. An integrated genomic analysis of human glioblastoma multiforme. Science 321, 1807-1812 (2008).

4 Yan H, Parsons DW, Jin G et al. IDH1 and IDH2 mutations in gliomas. N. Engl. J. Med. 360, 765-773 (2009).

5 Kloosterhof NK, Bralten LB, Dubbink HJ, French PJ, van den Bent MJ. Isocitrate dehydrogenase-1 mutations: 
a fundamentally new understanding of diffuse glioma? Lancet Oncol. 12, 83-91 (2011).

6 Watanabe T, Nobusawa S, Kleihues P, Ohgaki H. IDH1 mutations are early events in the development of astrocytomas and oligodendrogliomas. Am. J. Pathol. 174, 1149-1153 (2009).

7 Hartmann C, Meyer J, Balss J et al. Type and frequency of $I D H 1$ and $I D H 2$ mutations are related to astrocytic and oligodendroglial differentiation and age: a study of 1010 diffuse gliomas. Acta Neuropathol. 118, 469-474 (2009).

8 Takano S, Tian W, Matsuda M et al. Detection of IDH1 mutation in human gliomas: comparison of immunohistochemistry and sequencing. Brain Tumor Pathol. 28, 115-123 (2011).

9 Capper D, Simon M, Langhans CD et al. German Glioma Network. 2-Hydroxyglutarate concentration in serum from patients with gliomas does not correlate with $I D H 1 / 2$ mutation status or tumor size. Int. J. Cancer 131(3), 766-768 (2012).

10 SongTao Q, Lei Y, Si G et al. IDH mutations predict longer survival and response to temozolomide in secondary glioblastoma. Cancer Sci. 103(2), 269-273 (2012).

11 Seltzer MJ, Bennett BD, Joshi AD et al. Inhibition of glutaminase preferentially slows growth of glioma cells with mutant IDH1. Cancer Res. 70, 8981-8987 (2010).

12 Mardis ER, Ding L, Dooling DJ et al. Recurring mutations found by sequencing an acute myeloid leukemia genome. N. Engl. J. Med. 361, 58-66 (2009).

13 Cairns R, Iqbal Q, Lemonnier F et al. IDH2 mutations are frequent in angioimmunoblastic T-cell lymphoma. Blood 119, 1901-1903 (2012).

14 Amary MF, Bacsi K, Maggiani F et al. IDH1 and IDH2 mutations are frequent events in central chondrosarcoma and central and periosteal chondromas but not in other mesenchymal tumours. J. Pathol. 224, 334-343 (2011).

15 Borger D, Tanabe K, Fan KC et al. Frequent mutation of isocitrate dehydrogenase (IDH) 1 and $I D H 2$ in cholangiocarcinoma identified through broad-based tumor genotyping. Oncologist 17, 72-79 (2011).

16 Shibata T, Kokubu A, Miyamoto M, Sasajima Y, Yamazaki N. Mutant $I D H 1$ confers an in vivo growth in a melanoma cell line with BRAF mutation. Am. J. Pathol. 178, 1395-1402 (2011).

17 Ghiam AF, Cairns RA, Thoms J et al. IDH mutation status in prostate cancer. Oncogene 31, 3826 (2012).

18 Bleeker FE, Lamba S, Leenstra S et al. IDH1 mutations at residue p.R132 (IDH1(R132)) occur frequently in high-grade gliomas but not in other solid tumors. Hum. Mutat. 30, 7-11 (2009).

19 Ward P, Cross J, Lu C et al. Identification of additional IDH mutations associated with oncometabolite $\mathrm{R}(-)-2-$ hydroxyglutarate production. Oncogene 31(19), 2491-2498 (2012).

20 Reitman ZJ, Yan H. Isocitrate dehydrogenase 1 and 2 mutations in cancer: alterations at a crossroads of cellular metabolism. J. Natl Cancer Inst. 102, 932-941 (2010).

21 Joseph JW, Jensen MV, Ilkayeva O et al. The mitochondrial citrate/isocitrate carrier plays a regulatory role in glucose- stimulated insulin secretion. J. Biol. Chem. 281, 35624-35632 (2006).

22 Haselbeck RJ, McAlister-Henn L. Function and expression of yeast mitochondrial NAD- and NADP-specific isocitrate dehydrogenases. J. Biol. Chem. 268, 12116-12122 (1993).

23 Lee SH, Jo SH, Lee SM et al. Role of NADP+-dependent isocitrate dehydrogenase (NADP+-ICDH) on cellular defence against oxidative injury by gamma-rays. Int. J. Radiat. Biol. 80, 635-642 (2004).

24 Gupta R, Flanagan S, Li C et al. Expanding the spectrum of IDH1 mutations in gliomas. Mod. Pathol. 26, 619-625 (2013).

25 Pusch S, Sahm F, Meyer J, Mittelbronn M, Hartmann C, von Deimling A. Glioma IDH1 mutation patterns off the beaten track. Neuropathol. Appl. Neurobiol. 37(4), 428-430 (2011).

26 Ward P, Patel J, Wise DR, Abdel-Wahab O, Bennett BD et al. The common feature of leukemia-associated IDH1 and IDH2 mutations is a neomorphic enzyme activity converting alpha-ketoglutarate to 2-hydroxyglutarate. Cancer Cell 17, 225-234 (2010).

27 Dang L, White DW, Gross S et al. Cancer-associated IDHI mutations produce 2-hydroxyglutarate. Nature 462, 739-744 (2009).

- Demonstrated the accumulation of 2-hydroxyglutarate (2-HG) to be produced in tumors harboring mutations in $I D H 1$.

28 Gross S, Cairns RA, Minden MD et al. Cancer-associated metabolite 2-hydroxyglutarate accumulates in acute myelogenous leukemia with isocitrate dehydrogenase 1 and 2 mutations. J. Exp. Med. 207, 339-344 (2010).

- Demonstrated the accumulation of 2-HG in acute myelogenous leukemia as a result of mutations in $\mathrm{IDHI}$ and $I D H 2$.

$29 \mathrm{Xu}$ W, Yang H, Liu Y et al. Oncometabolite 2-hydroxyglutarate is a competitive inhibitor of alphaketoglutarate-dependent dioxygenases. Cancer Cell 19, 17-30 (2011).

- Demonstrated that accumulated 2-HG acts as an oncolmetabolite through the inhibition of $\alpha$-ketoglutarate-dependent enzymes.

30 Figueroa ME, Abdel-Wahab O, Lu C et al. Leukemic $I D H 1$ and IDH2 mutations result in a hypermethylation phenotype, disrupt TET2 function, and impair hematopoietic differentiation. Cancer Cell 18, 553-567 (2010).

31 Zhao S, Lin $\mathrm{Y}, \mathrm{Xu}$ W et al. Glioma-derived mutations in $I D H 1$ dominantly inhibit IDH1 catalytic activity and induce HIF-1 $\alpha$. Science 324, 261-265 (2009).

32 Sasaki M, Knobbe C, Itsumi M et al. D-2-hydroxyglutarate produced by mutant $I D H 1$ perturbs collagen maturation and basement membrane function. Genes Dev. 26, 2038-2049 (2012).

33 Chowdhury R, Yeoh KK, Tian YM et al. The oncometabolite 2-hydroxyglutarate inhibits histone lysine demethylases. EMBO Rep. 12, 463-469 (2011). 
34 Noushmehr H, Weisenberger DJ, Diefes K et al. Identification of a $\mathrm{CpG}$ island methylator phenotype that defines a distinct subgroup of glioma. Cancer Cell 17, 510-522 (2010).

35 Laffaire J, Everhard S, Idbaih A et al. Methylation profiling identifies 2 groups of gliomas according to their tumorigenesis. Neuro Oncol. 13, 84-98 (2011).

36 Christensen BC, Smith AA, Zheng S et al. DNA methylation, isocitrate dehydrogenase mutation, and survival in glioma. J. Natl Cancer Inst. 103, 143-153 (2011).

37 Lindahl G, Lindstedt G, Lindstedt S. Metabolism of 2-amino-5-hydroxyadipic acid in the rat. Arch. Biochem. Biophys. 119, 347-352 (1967).

38 Chalmers R, Lawson A, Watts R et al. D-2-hydroxyglutaric aciduria: case report and biochemical studies. J. Inherit. Metab. Dis. 3, 11-15 (1980).

39 Achouri Y, Noel G, Vertommen D, Rider MH, Veigada-Cunha M, Schaftingen E. Identification of a dehydrogenase acting on D-2-hydroxyglutarate. Biochem. J. 381, 35-42 (2004).

40 Struys EA, Verhoeven NM, Brink HJ, Wickenhagen WV, Gibson KM, Jakobs C. Kinetic characterization of human hydroxyacid-oxoacid transhydrogenase: relevance to D-2hydroxyglutaric and gamma-hydroxybutyric acidurias. J. Inherit. Metab. Dis. 28, 921-930 (2005).

- Demonstrates the kinnetics of the HOT enzyme and the relevance of abnormalities in hydroxyacid-oxoacid transhydrogenase enzyme function to pathological conditions.

41 Kaufman E, Nelson T, Fales H, Levin D. Isolation and characterization of a hydroxyacid-oxoacid transhydrogenase from rat kidney mitochondria. J. Biol. Chem. 263, 16872-16879 (1988).

42 Struys E. D-2-Hydroxyglutaric aciduria: unravelling the biochemical pathway and the genetic defect. J. Inherit. Dis. 29(1), 21-29 (2006).

43 Struys EA, Salomons GS, Achouri Y et al. Mutations in the D-2- hydroxyglutarate dehydrogenase gene cause D-2hydroxyglutaric aciduria. Am. J. Hum. Genet. 76, 358-360 (2005)

44 Struys EA, Verhoeven NM, ten Brink HJ, Wickenhagen WV, Gibson KM, Jakobs C. Kinetic characterisation of human hydroxyacid-oxoacid transhydrogenase: relevance to D-2- hydroxyglutaric and gamma-hydroxybutyric acidurias. J. Inherit. Metab. Dis. 28, 921-930 (2005).

45 Kranendijk M, Struys EA, Salomons GS, Van der Knaap MS, Jakobs C. Progress in understanding 2-hydroxyglutaric acidurias. J. Inherit. Metab. Dis. 35(4), 571-587 (2012).

46 Kranendijk M, Struys EA, van Schaftingen E. IDH2 mutations in patients with D-2-hydroxyglutaric aciduria. Science 330, 336 (2010).

47 Krell D, Assoku M, Galloway M, Mulholland P, Tomlinson I, Bardella C. Screen for $I D H 1, I D H 2, I D H 3$, $D 2 H G D H$ and $L 2 H G D H$ mutations in glioblastoma. PLoS ONE 6(5), e19868 (2011).
48 Brehmer S, Pusch S, Schmieder K, von Deimling A, Hartmann C. Mutational analysis of $D 2 H G D H$ and L2HGDH in brain tumors without $I D H 1$ or $I D H 2$ mutations. Neuropathol. Appl. Neurobiol. 37(3), 330-332 (2011).

49 Struys EA, Verhoeven NM, Brunengraber H, Jakobs C. Investigations by mass isotopomer analysis of the formation of D-2-hydroxyglutarate by cultured lymphoblasts from two patients with D-2-hydroxyglutaric aciduria. FEBS Lett. 557, 115-120 (2004).

50 Craigen WJ, Jakobs C, Sekul EA et al. D-2-Hydroxyglutaric aciduria in neonate with seizures and CNS dysfunction. Pediatr. Neurol. 10, 49-53 (1994).

51 Gibson KM, Craigen W, Herman GE et al. D-2-Hydroxyglutaric aciduria in a newborn with neurological abnormalities: a new neurometabolic disorder? J. Inherit. Metab. Dis. 16, 497-500 (1993).

52 Struys EA, Verhoeven NM, Salomons GS et al. D-2hydroxyglutaric aciduria in three patients with proven SSADH deficiency: genetic coincidence or a related biochemical epiphenomenon? Mol. Genet. Metab. 88, 53-57 (2006).

53 Kranendijk M, Struys EA, Gibson KM et al. Evidence for genetic heterogeneity in D-2-hydroxyglutaric aciduria. Hum. Mutat. 31(3), 279-283 (2010).

54 Struys EA, Verhoeven NM, Jansen EEW et al. Metabolism of $\gamma$-hydroxybutyrate to D-2-hydroxyglutarate in mammals: further evidence for D-2-hydroxyglutarate transhydrogenase. Metabolism 55(3), 353-358 (2006).

55 Al Aqeel A, Rashed M, Ozand PT et al. A new patient with alpha-ketoglutaric aciduria and progressive extrapyramidal tract disease. Brain Dev. 16(Suppl.), 33-37 (1994).

56 Kohlschutter A, Behbehani A, Langenbeck U et al. A familial progressive neurodegenerative disease with 2-oxoglutaric aciduria. Eur. J. Pediatr. 138, 32-37 (1982).

57 Terunuma A, Putluri N, Mishra P et al. MYC-driven accumulation of 2-hydroxyglutarate is associated with breast cancer prognosis. J. Clin. Invest. 124, 398-412 (2014).

-. Demonstrated that 2-HG accumulates in IDH wildtype breast tumors, driven by MYC, highlighting that abnormalities of gene function other than IDH can result in 2-HG accumultation an tumor development.

58 Natsumeda M, Igarashi $\mathrm{H}$, Nomura $\mathrm{T}$ et al. Accumulation of 2-hydroxyglutarate in gliomas correlates with survival: a study by 3.0-tesla magnetic resonance spectroscopy. Acta Neuropathol. Commun. 2, 158 (2014).

- Demonstrated that 2-HG accumulation occurs in gliomas which carry wild-type and not mutant $I D H$ suggesting that other processes may lead to 2-HG accumultation.

59 Walter MJ, Payton JE, Ries RE et al. Acquired copy number alterations in adult acute myeloid leukemia genomes. Proc. Natl Acad. Sci. USA 106(31), 12950-12955 (2009).

60 Tae CH, Ryu KJ, Kim SH et al. Alcohol dehydrogenase, iron containing, 1 promoter hypermethylation associated with colorectal cancer differentiation. BMC Cancer 13, 142 (2013). 
61

Popovici-Muller J, Saunders J, Salituro F et al. Discovery of the first potent inhibitors of mutant $I D H 1$ that lower tumor 2-HG in vivo. ACS Med. Chem. Lett. 3, 850 (2012).

62 Wang F, Travins J, DeLaBarre B. Targeted inhibition of mutant $I D H 2$ in leukemia cells induces cellular differentiation. Science 340(6132), 622-626 (2013).
63 Rohle D, Popovici-Muller J, Palaskas N et al. An inhibitor of mutant $I D H 1$ delays growth and promotes differentiation of glioma cells. Science 340, 626-630 (2013). 